\title{
Estudio de la Ergonomía de la Instrumentación de la Cabina de la DA20-C1 y Propuesta de Mejora
}

\section{Study of the Ergonomics of the DA20-C1 Cabin Instrumentation and Proposal for Improvement}

\author{
Andrés Dávalos Carrillo ${ }^{(1,2)}$, Allan Geovanny Cobo Vélez ${ }^{(1,2)}$ \\ (1) Universidad de las Fuerzas Armadas ESPE, Ecuador \\ (2) Fuerza Aérea Ecuatoriana F.A.E, Ecuador \\ andresdavalos79@gmail.com, coboallan@gmail.com
}

\begin{abstract}
Resumen
El presente estudio se enfoca en la importancia de la ergonomía de la instrumentación de la cabina de la DA20-C1 con el fin de mitigar problemas que causan la ausencia de la misma. El propósito principal de la presente investigación es determinar los problemas más relevantes de la ergonomía de esta cabina, para proponer la implementación del uso de un modelo CRM en las misiones de instrucción y operativas de la Escuela Superior Militar de Aviación "Cosme Rennella Barbatto" para reducir el agotamiento y el estrés del piloto. Este estudio de campo se desarrolla dentro de las instalaciones de la ESMA, incluyendo como población el personal de pilotos militares activos conformados de la siguiente manera: cadetes de la especialidad pilotos, pilotos operativos e instructores de vuelo. Para la obtención de los resultados, se verificó de modo directo las actividades que ellos cumplen. Adicional a eso, se realizaron encuestas para confirmar la existencia de los problemas ergonómicos de instrumentación cuando se encuentran en misiones reales de vuelo. Así mismo se verificó la viabilidad de implementar un modelo CRM ya que no se consideró viable el rediseño de aeronaves por su alto costo.
\end{abstract}

Palabras clave: Ergonomía, DA20-C1, modelo CRM, carga de trabajo.

\begin{abstract}
The present study focuses on the importance of the ergonomics of the DA20-C1 cockpit instrumentation in order to mitigate problems that cause its absence. The primary purpose of this research has been to determine the most relevant problems of the ergonomics of this cabin in order to propose the implementation of the use of a CRM model in the instruction and operational missions of the Escuela Superior Militar de Aviación "Cosme Rennella Barbatto" for reducing pilot exhaustion and stress. This field study has been conducted within the ESMA facilities, including active military pilot personnel, including cadets of the pilot specialty, operational pilots, and flight instructors. In order to obtain the results, the performed activities were directly verified. In addition, several surveys were performed in order to confirm ergonomic instrumentation problems during actual flight missions. Likewise, the viability of implementing a CRM model was verified since the redesign of aircraft was not considered feasible due to its high cost.
\end{abstract}

Keywords: Ergonomics, DA20-C1, CRM model, workload. 


\section{Introducción}

La Escuela Superior Militar de Aviación "Cosme Rennella Barbatto" (ESMA) ubicada en la península de Santa Elena, cantón Salinas, es una institución de educación militar que pertenece la Fuerza Aérea Ecuatoriana (FAE), donde se forman los futuros oficiales pilotos y técnicos de las diferentes especialidades (FAE, 2019).

Siendo en estas instalaciones donde se preparan a los futuros oficiales PILOTOS de la FAE que desempeñaran las diferentes funciones en el ámbito de la seguridad aeronáutica del país. En el año 1974, la FAE adquirió 24 avionetas Cessna Aerobat A-150L, para entrenamiento primario de los cadetes. Era una aeronave biplaza con tren de aterrizaje fijo tipo triciclo y con ala alta, contaba con un panel de instrumentos análogos para la navegación. Estas aeronaves fueron reemplazadas en el año 2012, al haber cumplido su tiempo de vida útil por los modernos equipos de vuelo DA20-C1 de la compañía Diamond Aircraft, posicionado como un excelente entrenador ligero, con bajos costos de mantenimiento y operatividad, y con una muy buena reputación en el ámbito de seguridad. Los mandos de la aeronave DA20 se basan en una palanca de control o bastón de mando, una carlinga de burbuja que ofrece gran visibilidad, a la baja y un depósito de combustible en la parte posterior de los asientos, con una cola en T para mayor estabilización (Aircraft, 2019).

En las misiones de instrucción y operativas en el equipo de vuelo DA20-C1, el piloto al mando de la aeronave debe situarse en el lado izquierdo de la cabina. En algunas fases se deben cumplir misiones utilizando los instrumentos de la cabina como en la fase de despegue y aterrizaje, vuelo de contacto y vuelo por instrumentos. Para ello el piloto es el encargado de realizar todos los procedimientos y chequeos, en el caso del piloto alumno sin ayuda del instructor. En el despegue y aterrizaje el piloto debe maniobrar con la mano izquierda el bastón de mando y con la derecha prender y apagar los switches de las luces, estos además son todos de color blanco, En este movimiento de manos se genera una incomodidad para maniobrar la avioneta. También existe molestia al utilizar ciertos instrumentos que se encuentran en el lado derecho de la cabina para el piloto al mando, ya que esto implica que tiene que desplazar el cuerpo para alcanzarlos. Por otra parte, los pilotos en la ESMA deben utilizar guantes de vuelo, y al ser botones pequeños y del mismo color ciertos instrumentos, muchas veces hace que provoque errores al utilizarlos ya que da la sensación de que se está pulsando otros.

Estos problemas son los que generan incomodidad al utilizar los instrumentos de navegación y a su vez generan estrés, como la posición alejada del piloto al mando y que algunos sean del mismo color, estén muy continuos, y sean muy pequeños, provoca dificultad en el control de los instrumentos con el uso de guantes de vuelo.

Para realizar un vuelo seguro es necesario que los pilotos tengan una buena ergonomía en el uso de los instrumentos de la cabina de la DA20-C1, y en algunos casos existe la incomodidad al utilizar estos instrumentos, al no poder hacer una modificación física de la cabina para mejorar este problema, y en misiones de instrucción donde el alumno es quien debe realizar toda la carga de trabajo sin ayuda del instructor, se debe aplicar un método factible para reducir esta carga de trabajo y generar menos estrés en el piloto. Por ello esta investigación busca mitigar el agotamiento y el stress del piloto causado por la incomodidad que generan ciertos instrumentos por su posición, tamaño y color. Y así lograr que se beneficien los aspirantes a pilotos de la ESMA disminuyendo el estrés en las misiones de instrucción y operación. En estas misiones de vuelo se aplicará un modelo CRM (Crew Resource Management) entre ambos pilotos para evitar la pérdida de la conciencia situacional cuando se genere estrés por los 
problemas ergonómicos de los instrumentos. Para misiones de instrucción se desarrollará un modelo de vuelo imaginario para ayudar al alumno a volar con más seguridad, para misiones operativas, un modelo de ayuda mutua entre ambos pilotos en cabina para reducir la carga de trabajo del piloto al mando. Se pretende que, con la aplicación correcta del CRM, ayude a un entrenamiento con menor estrés psicológico por la carga de trabajo, ubicación, tamaño y color de los instrumentos de navegación, que genera problemas ergonómicos en el piloto alumno.

\section{Materiales y Métodos}

\subsection{Ergonomía}

\section{Definición de Ergonomía}

La palabra ergonomía nace de los términos griegos: ergon (trabajo) y nomos (ley o norma) que significaría las normas para trabajar" (Quiñonez, 2018).

Sin embargo, la utilización de este término como la adaptación de una persona a las condiciones de trabajo se debe a Murrell, con la creación de la primera sociedad de ergonomía en julio de 1949, la llamada "Ergonomics Research Society", fundada por británicos expertos en fisiología y psicología con el fin de adaptar el trabajo al hombre y no viceversa (Quiñonez, 2018).

\subsection{Interfaz Persona-Máquina}

\section{Sistemas Manuales}

La principal particularidad de este sistema es la persona quien aporta su propia energía para el funcionamiento del mismo, y que la intervención que ejecuta sobre los resultados es directa, es decir, los errores van directamente relacionados con el factor humano. (Mondelo, Gregori, \& Barrau, 1994, pág. 29).

\section{Sistemas mecánicos}

Son sistemas en la cual la persona recibe la información de cómo funciona la maquina a través de dispositivos informativos y sus decisiones son las que regulan el funcionamiento de esta (Mondelo, Gregori, \& Barrau, 1994, pág. 29).

\section{Dispositivos informativos (DI)}

La necesidad de obtener información es indispensable para que el piloto controle la aeronave; la retroalimentación que recibirá, la cantidad y calidad de los datos, su cadencia, la forma en que la recibe, etc.... determinarán la calidad de la respuesta del piloto frente a las situaciones que se le presenten en las misiones de vuelo. De acuerdo con los canales que se recibe la información, la visión es el sistema de recepción en el cual el piloto recibe más del $80 \%$ de datos y el restante proveniente de la audición y del tacto (Mondelo, Gregori, \& Barrau, 1994, pág. 32).

\section{Dispositivos informativos visuales (DIV)}

La contrariedad de los indicadores visuales radica en que no sólo obedecen de la perspicacia 
visual del piloto, sino que además se debe considerar las condiciones externas que configuran el espacio de la cabina, y que interfieren en el procesamiento de los datos visuales. Los fabricantes de aeronaves deben elegir un dispositivo que, cumpliendo los requisitos legales de aeronavegabilidad, sea el más sencillo de todos. Por ello se establecen los siguientes parámetros de acuerdo con (Mondelo, Gregori, \& Barrau, 1994, pág. 33):

- Visibilidad: brillo y contraste

- Legibilidad: tamaño, claridad y tipo de fuente luminosa

- Grado de fatiga: fuente luminosa, color, parpadeo

- Compatibilidad: grado de adaptación del sistema

A continuación, se enlista los DIV básicos:

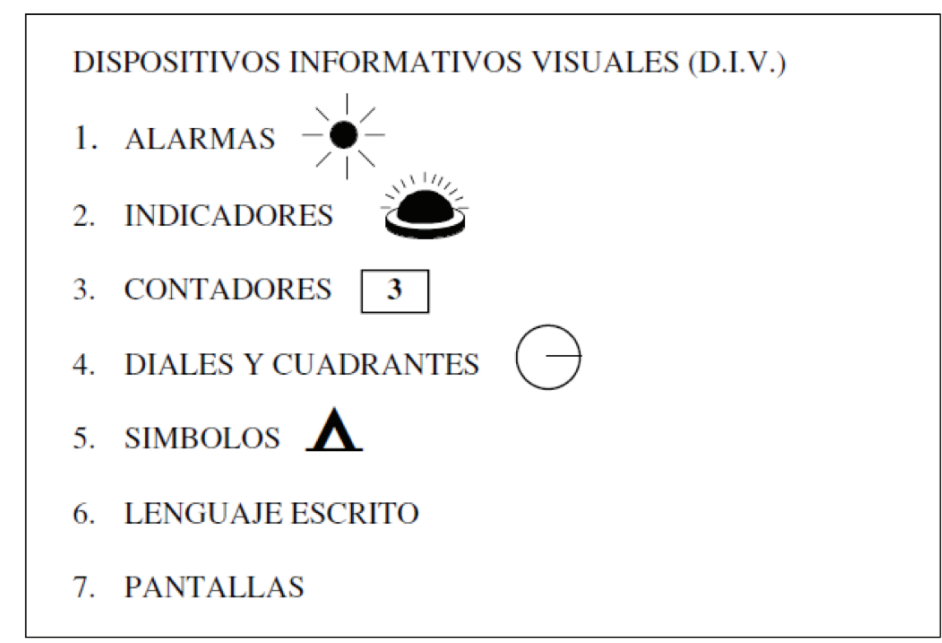

Figura 1: DIV básicos.

Fuente: CITATION Mon94 \1 12298 (Mondelo, Gregori, \& Barrau, 1994)

\subsection{Relaciones de Control Entre Hombre-Instrumentos}

El control de los instrumentos es el objetivo final del piloto, todo este sistema tiene que proyectarse para que su confianza este dentro de los limites apropiados, para ellos se debe captar la información de los DI en tal forma que sea significativa y contribuya a las misiones de vuelo. Para ello es indispensable que exista una secuencia de interacciones entre las relaciones informativas y las relaciones dimensionales. Para que exista una buena ergonomía en el uso de los instrumentos de navegación, estos deben cumplir las siguientes funciones básicas que no generen incomodidad al momento de utilizarlos (Mondelo, Gregori, \& Barrau, 1994, pág. 47):

- Activar y desactivar las luces

- Fijación de valores discretos (los indicadores deben mostrar escalas pequeñas)

- Fijación continua de valores (control de volumen de las comunicaciones en un solo sector)

- Control ininterrumpido (bastón de mando)

- Entrada de datos (Fijar los cursos y coordenadas)

En la Figura 2 se muestra un ejemplo de mandos utilizados en la aviación estadounidense: 

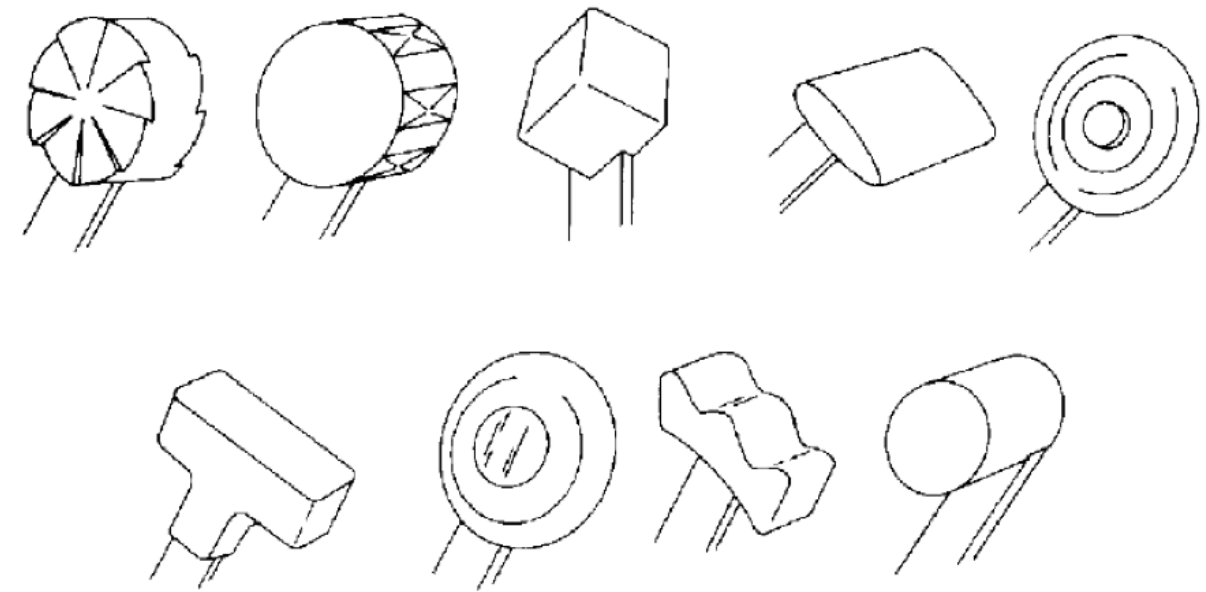

Figura 2: Mandos de forma codificada y estandarizada que emplean los aviones de la United States Air Force Fuente: CITATION Mon94 $\backslash 12298$ (Mondelo, Gregori, \& Barrau, 1994)

\section{Compatibilidad espacial}

El piloto debe optimizar el uso de los instrumentos de navegación, para ello el panel debe estar diseñado de tal manera de que permita atender a las siguientes ideas básicas:

- Un entrenamiento y aprendizaje más eficiente y eficaz

- $\quad$ Reducir el número de accidentes

- Mejores respuestas ante sobrecarga de trabajo y estrés

- Mejor rapidez y precisión en el uso de instrumentos

\subsection{Ergonomía en la Aviación}

Como ya se ha tratado en esta investigación, la ergonomía está muy conexa con la aviación, ya que esta ciencia está presente en el diseño de aeronaves. La aviación es un sistema de relación entre hombre-aeronave que debe brindar todas las facilidades para hacer de un vuelo seguro, eficiente y eficaz al mismo tiempo. Sin embargo, históricamente la ergonomía era un tema alejado en la aviación militar, hasta que las autoridades se dieron cuenta de que la mayoría de los accidentes aeronáuticos estaban relacionados con errores de diseño de las interfaces de la cabina del piloto o a su vez con la fatiga que produce la carga de trabajo y estrés de los operadores.

La ergonomía surge de la necesidad de mejorar los recursos externos que se le brindan al piloto de la aeronave, es decir, adaptar la máquina al hombre, de ahí nace la idea de integrar en el mundo los diseños de las interfaces informáticas o simbólicas en la organización de los recursos de la cabina, y también la capacitación hacia los pilotos para que saquen la mayor eficacia en el uso de los instrumentos de navegación. Quizás por esto, es más difícil hablar de ergonomía de una aeronave sabiendo que no todas las personas reúnen las mismas capacidades fisiológicas o psicológicas, y aparece el llamado CRM, que se enfoca en la adaptación del piloto en los recursos de la cabina, lo que sería una solución más viable con menos costo al de hacer cambios en los sistemas continuamente. Analizado del artículo de la revista del COPAC (Aslanides, 2006, pág. 10). 


\section{Avioneta DA20-C1}

\section{Instrumentación de la cabina}

Los instrumentos de vuelo son los que se instalan en el lado piloto al mando.

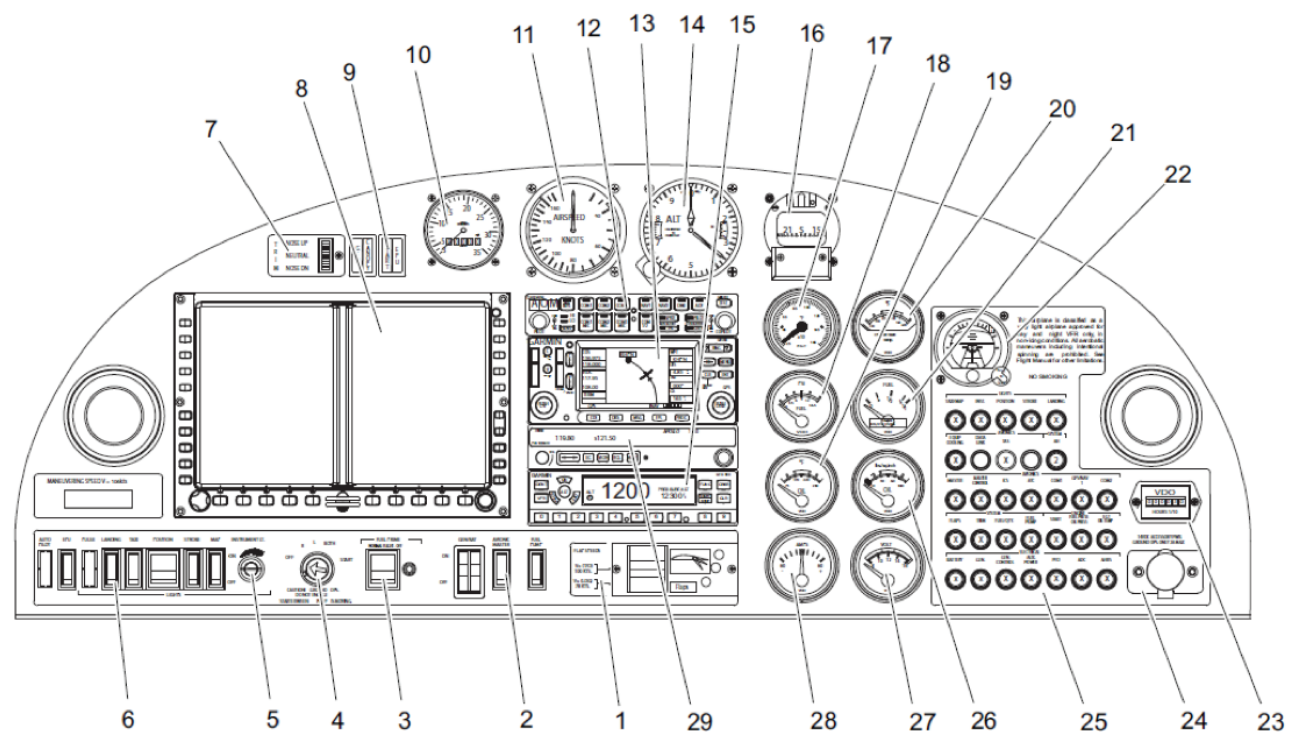

Figura 4: Panel de instrumentos

Fuente: (Aircraft, 2019, pág. 337)

1. Flap speeds

2. Panel de interruptores maestros
a. Aviónica Máster
b. Switch de bomba de combustible
c. Switch GEN/BAT

3. Combustible primario

4. Switch de ignición

5. Switch de instrumentos de luces

6. Switch de panel de luces
a. MAP
b. Switch de luz STROBE
c. Posición
d. Switch de luz TAXI
e. Switch de luz LANDING
f. Optional switch
g. EPU
h. PITOT
i. Auto Pilot 
7. Indicador Trim

8. Pantalla GDU 620

9. Luces de emergencia

10. Revoluciones del motor

11. Indicador de velocidad del aire

12. Selector automático

13. Navegación/Comunicación GPS

14. Altímetro

15. Comunicaciones VHF

16. Brújula magnética

17. Temperatura del gas de escape

18. Indicador de presión del combustible

19. Temperatura de la cabeza del cilindro

20. Indicador de temperatura del aceite

21. Indicador de contenido de combustible

22. Indicador de horizonte artificial (no disponible)

23. Horómetro Hobbs

24. VDC Aux Power Outlet

25. Panel Ciurt-Breaker

26. Indicador de presión del aceite

27. Voltímetro

28. Amperímetro

29. SL40 (COM 2)

\subsection{Misiones de Instrucción y Operativas}

Las misiones de instrucción se diferencian de las operativas porque en la primera se tiene como piloto al mando al cadete alumno, quien es el que debe realizar todos los procedimientos para cumplir con la misión de vuelo y se evidenció que a este es quien le afecta más los problemas ergonómicos. Y el piloto operativo que tiene más flexibilidad en compartir la carga de trabaja con la otra persona, pudiendo así reducir el estrés causado por dichos problemas de ergonomía. Todo esto de acuerdo con (FAE, Manual de Fase del equipo DA20 C-1, 2018):

\section{Fase de contacto}

El objetivo de esta fase es describir los parámetros, procedimientos y técnicas para ejecutar las diferentes maniobras en vuelo de contacto, a fin de estandarizarlas, respetando los límites de operación del avión; así como también, familiarizar al piloto con los procedimientos Operativos Normales aplicables al equipo de vuelo DA20C-1. 
Antes de iniciar cada una de las maniobras, la tripulación debe observar las condiciones que establecen los CHEQUEOS PARA MANIOBRAS:

1) THROTTLE

2) MIXTURE

3) FUEL

4) FLAPS

5) VELOCIDAD

6) ALTURA

7) ZONA DE TRABAJO

8) OBJETOS SUELTOS
AS REQUIERED

FULL RICH

PUMP ON

CRUISE

100 KIAS

$3000 \mathrm{fts}$. MINIMUM and set in the G500

CHECK

CHECK

\section{Despegue}

El despegue es el movimiento del avión desde su punto de inicio hasta que deja la tierra en vuelo controlado. Con el avión alineado en el eje de la pista, se realiza los procedimientos de despegue, el piloto suelta los frenos e incrementa la potencia despacio y de manera continua, aproximadamente a los 1000 pies de recorrido en la pista en la carrera de despegue sobre el nivel del mar, el piloto obtiene 44 KIAS, con esta velocidad realiza la rotación de la aeronave y mantiene una actitud positiva de despegue (Air Comet Virtual, 2014)

\section{Ascenso}

Es una maniobra básica durante la cual una combinación adecuada de potencia y actitud que permite ganar altura al avión. El ascenso se realiza con 75 KIAS, teniendo como referencia visual el panel de instrumentos sobre el horizonte natural. La tripulación, debe mantener durante el ascenso las manos sobre los respectivos controles de vuelo y potencia (bastón y acelerador) (Air Comet Virtual, 2014)

\section{Aterrizaje}

Esta maniobra se ejecuta al finalizar la misión de vuelo. El piloto ha finalizado los objetivos de la misión o ya ha cumplido con el tiempo previsto de vuelo y el piloto no tiene intención de volver al aire. (Air Comet Virtual, 2014)

\section{Fase de instrumentos}

El vuelo por instrumentos es una parte muy importante dentro de la formación del piloto de la ESMA, pues de la misma forma que en el vuelo por contacto el piloto alumno controla la aeronave en base a referencias geográficas y con el horizonte natural, dentro del vuelo instrumental se controla la aeronave utilizando de forma inmediata todos los instrumentos de control y comportamiento que están a bordo.

\subsection{Problemas Ergonómicos de Instrumentación de la Cabina de la DA20-C1}

La figura 5, es una foto real de la instrumentación de la cabina de la DA20-C1 en la cual se analizó todos los problemas ergonómicos que puede presentar el piloto al mando de la aeronave en las fases de despegue y aterrizaje, así como en misiones controladas por instrumentos. 


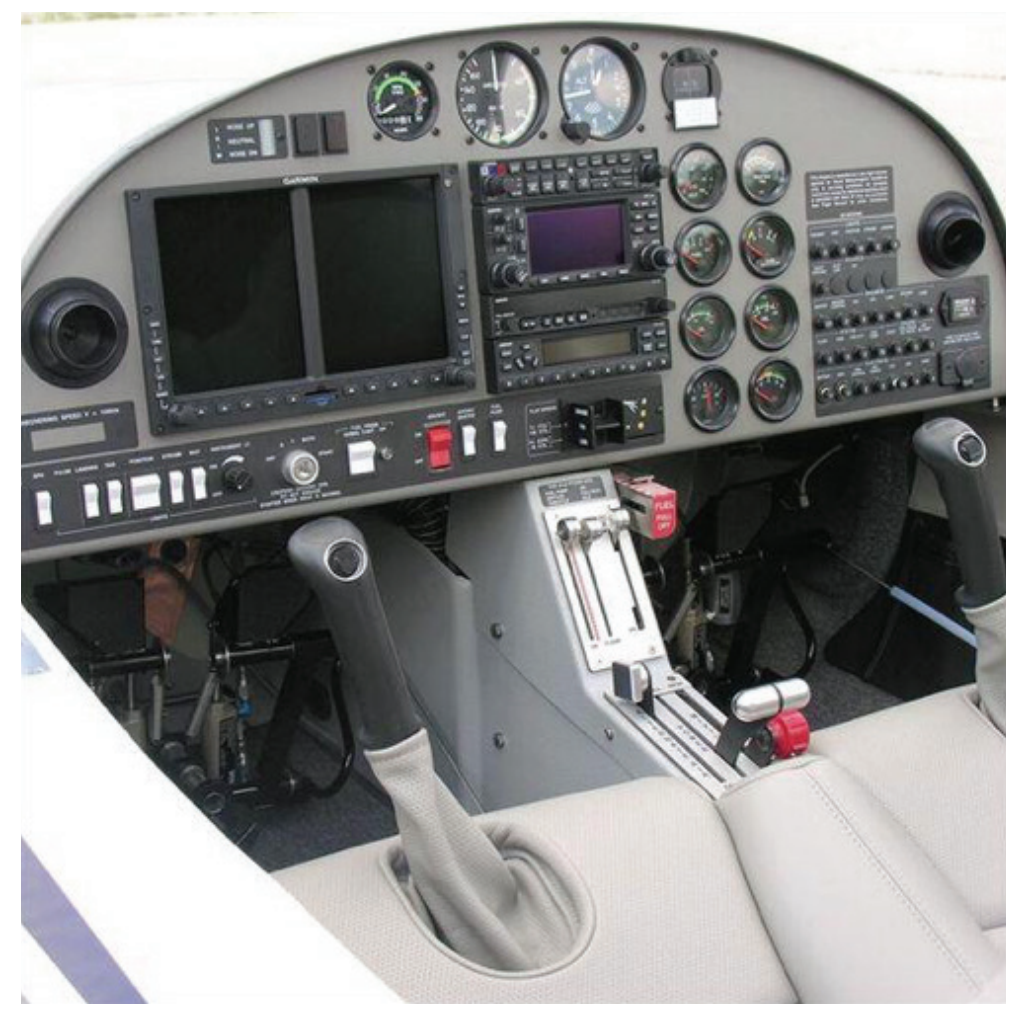

Figura 5. Instrumentos de la cabina de la DA20-C1.

\section{Posición de instrumentos de navegación}

El piloto al mando es quien se encuentra al lado izquierdo de los asientos "side by side" y tiene el control de la aeronave mediante el uso de las pantallas PDF y la mayoría de los instrumentos de navegación. Un tema incomodidad para el piloto es que cuando este requiere utilizar o hacer cambios en el panel Circuit-Breaker que se encuentra a su lado derecho, este tendría que estirarse para alcanzarlos tomando en cuenta que el otro piloto se encuentra a su lado derecho; así mismo, misiones en donde el instructor decide apagar la pantalla PDF para que el piloto alumno utilice solo los instrumentos análogos, generan incomodidad estar pendiente de muchos indicadores a la vez, como en misiones de fase de instrumentos. Todos estos son puntos que se suman a la carga de trabajo sobre el piloto alumno, quien es el que realiza la mayoría de todos los procedimientos establecidos para las diferentes maniobras.

\section{El tamaño, continuidad y color de los switches}

Los botones de los instrumentos como el auto selector, navegación/comunicación GPS, comunicaciones VHF y COM 2, son de color negro y se confunden con el fondo del panel, además que se encuentran todos en continuidad y poseen el mismo tamaño, hacen parecer a simple vista que todos son iguales, sin embargo, cada uno sirve para una función diferente, por ello el piloto al momento de pulsarlos debe tener cuidado de no haber presionado otro, esto a la par de estar verificando los demás instrumentos para no perder el curso de la aeronave. Contando que los pilotos deben usar obligatoriamente guantes de vuelo, que en ciertos casos son un poco más grandes de la medida de la mano del piloto, provoca errores e implica que se tenga que volver a chequear 
para comprobar si se ingresaron los datos correctos. Esto genera un pequeño estrés a la larga en las misiones donde se utilizan muy seguido estos instrumentos de navegación y comunicación, añadiendo la carga de trabajo que realiza el piloto al mando.

\section{Encender o apagar luces en el despegue y aterrizaje}

En el despegue y aterrizaje se necesita hacer procedimientos para encender y apagar las luces, por ello el piloto al mando con su mano izquierda maniobra la aeronave a través del bastón de mando y con su mano derecha utilizar los switches de las luces, debido a la posición del panel de luces provoca que el piloto tenga que cruzar los brazos. Esto genera en ocasiones que el piloto realice movimientos involuntarios en la aeronave, es decir, un leve movimiento en el bastón de mando podría provocar un cabeceo o alabeo de esta, que se traduce en un pequeño factor de peligro principalmente en los aterrizajes en donde se requiere la mayor concentración posible al ser la maniobra más difícil de todo el vuelo. Esto sin duda se considera un problema ergonómico de la instrumentación de la cabina de la DA20-C1 que se ha podido encontrar en esta investigación. Sin embargo, las falencias en los sistemas o interfaces hombre-máquina deben ser resueltas por ergónomos que trabajan en los diseños de las aeronaves. Estos expertos son los que trabajan pensando en la comodidad del piloto. Por ello la mejor forma de tratar estos inconvenientes y más viable considerando los gastos económicos de hacer una modificación a una aeronave los cuales son muy costosos, es aplicar técnicas de CRM para mejorar las habilidades de comunicación, manejo adecuado de los instrumentos de la cabina y soporte entre ambos pilotos para reducir la carga de trabajo y estrés del piloto.

\section{Carga de trabajo y estrés del piloto}

Principalmente en misiones de instrucción la carga de trabajo va toda hacia el piloto alumno, es decir, este es quien debe realizar todos los procedimientos para los diferentes tipos de misiones, ya que como parte de su formación como piloto debe obtener las capacidades y conocimientos para que pueda tomar el mando de la aeronave sin la ayuda de otro piloto. También podemos definir esta carga de trabajo como una carga mental, ya que no se requiere un esfuerzo físico mayor, sino más bien el uso de reglas y procedimientos estrictamente aplicados. Por ello la carga mental se refiere a toda la información que tiene recibir una persona, el tiempo que dispone para procesarla y la importancia que conlleva tomar una decisión. Por ejemplo, en el caso de una misión de instrucción donde el piloto alumno tiene una carga mental mayor porque sus decisiones implican una nota en sus misiones de vuelo y de eso depende si aprueba o no un curso de vuelo; a diferencia de una misión operativa donde existe más flexibilidad en la comunicación y se puede compartir la carga de trabajo entre ambos pilotos.

\section{CREW Resource Management en la Aviación (CRM)}

El CRM fue creado para evitar o reducir errores en las tripulaciones aéreas (Wiener, Kanki y Helmreich, 1993), este método se define como la mejor forma de utilizar los recursos disponibles de la cabina por parte de los pilotos, como pueden ser las pantallas, sistemas GPS, comunicaciones e inclusive las habilidades de la misma tripulación, para garantizar condiciones seguras de vuelo.

El objetivo principal del CRM es utilizar las capacidades tanto de la aeronave como del piloto para que las misiones de vuelo sean más eficientes y eficaces (Federal Aviation Administration [F.A.A.], 2012). 


\subsection{Metodología de la Investigación}

La modalidad del presente trabajo es de campo con enfoque mixto, se realizó visitas a los hangares de vuelo donde se encuentra la aeronave DA20-C1 para la observación directa, entrevistas y encuestas a los instructores pilotos y únicamente encuesta a los alumnos que se entrenan como oficiales pilotos, en dichas encuestas se consideran puntos como las especificaciones de las misiones de vuelo en que utilizan los instrumentos de navegación. Evidenciando la necesidad de implantar el uso de CRM para reducir el estrés psicológico que afecta al uso de los instrumentos de navegación, por la incomodidad que existe en la ubicación de ciertos switches y breakers.

\subsection{Técnicas e Instrumentos de Investigación}

Se realizó la observación directa donde se pudo analizar todos los aspectos que se realizan en una misión de vuelo con respecto al uso de los instrumentos de navegación, para evidenciar que problemas ergonómicos presenta el piloto alumno al utilizar los switches, breakers y pantalla de navegación.

Se realizó una encuesta con 8 preguntas y con 3 escalas de posibles respuestas como: si, no, talvez, dichas preguntas fueron basadas en el resultado de la observación directa en los hangares antes de las misiones de vuelo de los cadetes pilotos, oficiales instructores y operativos de la ESMA sobre los problemas más comunes que se evidenciaron de ergonomía con respecto al uso de la instrumentación de la cabina de la DA20-C1 para poder analizar un modelo que ayude a mejorar estos inconvenientes.

La población objetivo fueron los cadetes pilotos, personal de oficiales e instructores de vuelo, ya que son los que realizan las misiones de vuelo donde utilizan los instrumentos de navegación de la cabina de la DA20-C1. Se seleccionó una muestra representativa al azar de la población de cadetes pilotos, personal de oficiales e instructores de vuelo.

Tabla 1: Población y muestra (ESMA, 2019)

\begin{tabular}{ccc}
\hline DESCRIPCIÓN & POBLACIÓN & MUESTRA \\
\hline Cadetes pilotos & 31 & 31 \\
\hline Oficiales pilotos & 20 & 15 \\
\hline Oficiales Instructores & 12 & 4 \\
\hline Total & 63 & 50 \\
\hline
\end{tabular}

Fuente: ESMA 2019 


\section{Evaluación de Resultados y Discusión}

\subsection{Resultados Generales}

En relación con los resultados generales obtenidos en la investigación se demostró que sí existen problemas ergonómicos con respecto al uso de los instrumentos de navegación de la cabina de la DA20-C1, tales como: la posición de instrumentos, tamaño, continuidad y color de switches, sensibilidad de perillas y selectores, incomodad de manipular el curse, Heading o Alímetro desde la cabina izquierda.

Tabla 2: Resultados (ESMA, 2019)

\section{PREGUNTA}

¿La posición de ciertos instrumentos de navegación causa incomodidad para el piloto?

$60 \% \quad 32 \% \quad 8 \%$

¿El tamaño, continuidad y color de switches provoca errores al pulsar los instrumentos?

$64 \% \quad 28 \% \quad 8 \%$

¿Al momento de encender y apagar las luces en el despegue y aterrizaje siente incomodidad?

$64 \% \quad 32 \% \quad 4 \%$

¿En las misiones operativas existe más flexibilidad en la comunicación entre ambos pilotos?

$56 \% \quad 12 \% \quad 32 \%$

¿Sabe usted lo que significa CRM?

$44 \% \quad 52 \%$

$4 \%$

¿Cree usted que el uso de CRM en misiones de vuelo reduce la carga de trabajo y estrés del piloto?

$64 \% \quad 32 \% \quad 4 \%$

¿Le causa estrés la sensibilidad de las perillas y selectores al momento de ser manipulados?

$66 \% \quad 28 \% \quad 6 \%$

¿Durante un vuelo instrumental en "cabina izquierda" siente incomodidad al manipular el Curse, Heading o Altímetro?

\section{Fuente: ESMA 2019}

De acuerdo con las entrevistas realizadas se determinó que la carga de trabajo en misiones de instrucción en el piloto alumno es mayor frente a oficiales pilotos operativos causando cansancio psicológico y stress. Entre los problemas más destacados también se mostró que la posición de ciertos switches causa incomodidad en el piloto, el parecido de ciertos botones que se encuentran en 
el mismo sector provoca que el piloto erróneamente pulse otro y este tenga que verificar varias veces si ha ingresado los datos correctos. También al momento de encender o apagar las luces en el despegue y aterrizaje genera movimientos involuntarios de la aeronave. Por ello es necesario que implemente un modelo CRM para misiones de instrucción y operativas tomando en cuenta las diferencias que existen entre ambas. Con este estudio se espera que aplique un modelo CRM en misiones operativas y de instrucción, que puede definirse como un sistema de gestión que hace un uso óptimo de todos los recursos disponibles (equipos, procedimientos y personas) para promover la seguridad y mejorar la eficiencia de las operaciones de vuelo. Por ello, para misiones se implementará el trabajo en equipo, mientras un piloto necesite estar más cómodo para manipular los instrumentos puede ceder el mando del avión al otro piloto, para que así este pueda pulsar correctamente los switches sin causar movimientos involuntarios en la aeronave. También de que se solicite al otro piloto que manipule ciertos instrumentos mientras el revisa la pantalla PFD. Promover una mejora en las habilidades de comunicación entre ambos pilotos, es decir, que entre ambos se recuerden los procedimientos antes de manipular los instrumentos, que se visualice bien los switches para que no haya confusión al momento de pulsarlos. Y para misiones de instrucción implementar el vuelo imaginario como parte de un CRM para el manejo adecuado de los instrumentos de la cabina, es decir, que se repase todos los procedimientos que debe realizar el piloto alumno en el aire, de forma sistemática y que se incluya en el plan de clases para los alumnos como respuesta a estos problemas ergonómicos. Estos dos modelos CRM ayudará a reducir el estrés por la carga de trabajo tanto para un piloto operativo como para un piloto alumno.

\subsection{Propuesta de Mejora}

\section{Misiones de instrucción}

Las misiones de instrucción son aquellas donde existe la relación instructor-alumno y este último es quien está al mando de la aeronave. Frente a los problemas ergonómicos de la instrumentación de la cabina DA20-C1 encontrados en esta investigación, se determinó que es en estos tipos de misiones en donde afectan más al piloto alumno por la carga de trabajo que este debe soportar.

La implementación de vuelo imaginario dentro de la cabina en horarios establecidos para ayudar a contrarrestar los problemas ergonómicos hallados en esta investigación.

- Observar repetitivamente donde se encuentra ubicado cada instrumento de la cabina para que el piloto encuentre cual es la mejor forma de alcanzarlos para que pueda manipularlos sin moverse en posiciones incómodas.

- Recrear misiones dentro de la cabina donde se utilice los botones de los instrumentos como el auto selector, navegación/comunicación GPS, comunicaciones VHF y COM 2 para que el piloto alumno disminuya la probabilidad de cometer errores en el aire.

- Repasar continuamente en vuelos imaginarios los procedimientos para encender y apagar las luces en el despegue y aterrizaje para que el piloto alumno se acostumbre a cruzar los brazos en esta maniobra y evitar movimientos involuntarios de la aeronave.

Todos estos vuelos imaginarios tienen que ser supervisados por un instructor y desarrollados en horas de clases como parte de la formación de vuelo. 


\section{Misiones Operativas}

La diferencia de estos tipos de misiones es que en esta vuelan la aeronave dos pilotos operativos, esto implica que la carga de trabajo si puede dividirse para ambos pilotos y el estrés puede reducirse en comparación a la de un piloto alumno. La propuesta de mejora es implementar un modelo CRM para fortalecer las habilidades de comunicación dentro de la cabina y hacer un mejor uso de los recursos disponibles.

- Desarrollar el trabajo en equipo dentro de las misiones operativas, si cualquiera de los dos pilotos siente incomodidad de utilizar algún switch o perilla, solicitarle al otro piloto que lo haga por él.

- Cuando se realice procedimientos para ingresar datos en el curse o en el GPS, el piloto más antiguo le dictará la información al menos antigua para que este los ingrese y así poder reducir la carga de trabajo.

- Al momento de encender las luces, el copiloto tome el mando de la aeronave durante un breve lapso para que el piloto pueda manipular los switches cómodamente sin preocupación de que genere movimientos involuntarios en la aeronave.

\section{Conclusiones}

La investigación concluyó que existen 5 problemas de ergonomía con respecto al uso de varios instrumentos de navegación de la aeronave DA20-C1, tales como: la posición de instrumentos, tamaño, continuidad y color de switches, sensibilidad de perillas y selectores, incomodad de manipular el curse, Heading o Alímetro desde la cabina izquierda, lo que permite proponer mejoras para mitigar el cansancio y estrés del piloto, por lo que aplicar un modelo CRM de vuelo imaginario en misiones de instrucción en la aeronave DA20-C1 contribuye a un manejo más adecuado de los instrumentos de cabina para reducir los errores en el aire por parte del piloto alumno.

Aplicar un modelo CRM en misiones operativas en la aeronave DA20-C1 contribuye a reducir la carga de trabajo para el piloto al mando y optimizar la seguridad en vuelo gestionando de mejor forma los recursos humanos y mecánicos de la cabina.

Dentro de la escuela no se cuenta con los recursos económicos para financiar la compra, mejora o cambios en los equipos de la cabina de la avioneta DA20C, pero no es necesario del recurso económico para la viabilidad de esta investigación porque la ESMA cuenta con los recursos materiales como: hangares donde se encuentran las avionetas disponibles para su estudio, los pilotos instructores y alumnos que ayudaron al estudio y poder proponer soluciones para la mejora de la ergonomía con los instrumentos de la cabina, en la ESMA nos han facilitado con el tiempo para poder realizar la investigación con la debida autorización del Departamento de entrenamiento aéreo (DEA), Departamento cuerpo de cadetes y Departamento académico.

El fin de mejorar la comodidad del piloto al mando de la aeronave, rechazar procedimientos que le resulten incomodos y frente a ellos buscar ayuda con un proceso de CRM en misiones operativas e implementar un modelo estructurado de vuelo imaginario en misiones de instrucción para mejorar el rendimiento en la aeronave con el uso de los instrumentos de navegación y reducir la carga de trabajo ayudándolo a sentirse más seguro al momento de volar. 


\section{Referencias bibliográficas}

Air Comet Virtual. (15 de noviembre de 2014). ISSUU. Obtenido de https://issuu.com/airlevante/docs/ jose_-_reglas_basicas_vfr

Aircraft, D. (16 de octubre de 2019). DA20-C1. Obtenido de https://www.diamondaircraft.com/en/flightschool-solution/aircraft/da20/tech-specs/

Asamblea Nacional Del Ecuador. (2010). Ley Orgánica de Educación Superior. Quito: Registro Oficial 298.

Aslanides, M. (2006). Ergonomía y aviación: Matrimonio de conveniencia. Revista del COPAC, 10-12.

ESMA. (2019). Encuestas aplicadas. Salinas.

FAE. (2018). Manual de Fase del equipo DA20 C-1. Quito: Comando de Educación y Doctrina Aeroespacial COED.

FAE. (16 de octubre de 2019). Fuerza Aérea Ecuatoriana. Obtenido de https://reclutamientofae.mil.ec/ index.php/escuelas-militares/escuela-superior-militar-de-aviacion/quienes-somos

Mondelo, P. R., Gregori, E., \& Barrau, P. (1994). Ergonomía 1 Fundamentos. Barcelona, España: Mutua Universal.

Quiñonez, R. E. (septiembre de 2018). Plan de mejora de riesgos ergonómicos en el área de logística de una planta de fabricación de cajas de cartón corrugado y micro corrugado en la ciudad de Guayaquil. Guayaquil, Guayas, Ecuador.

Wiener, E. Kanki, B. y Helmreich, R. (Eds.) (1993). Manejo de los recursos de la cabina. San Diego, CA: Academic Press.

Lauber, J. K. (1984). Recursos de la cabina. Air Line Pilot, 53, 20-30.

Federal Aviation Administration (2012). Historia del CRM [Video]. Washington, DC: F.A.A. TV 